\title{
Influence of the distribution of the properties of permanent magnets on the field homogeneity of magnet assemblies for mobile NMR
}

\author{
Y.P.Klein ${ }^{1 *}$, L.Abelmann ${ }^{1,2}$, and J.G.E.Gardeniers ${ }^{1 *}$ \\ ${ }^{1}$ University of Twente, Enschede, The Netherlands \\ ${ }^{2}$ KIST Europe, Saarbrücken, Germany
}

\begin{abstract}
We optimised the magnetic field homogeneity of two canonical designs for mobile microfluidic NMR applications: two parallel magnets with an air gap and modified Halbach array. Along with the influence of the sample length, general design guidelines will be presented. For a fair comparison the sensitive length of the sample has been chosen to be the same as the gap size between the magnets to ensure enough space for the transmitting and receiving unit, as well as basic electric shimming components. Keeping the compactness of the final device in mind, a box with an edge length 5 times the gap size has been defined, in which the complete magnet configuration should fit. With the chosen boundary conditions, the simple parallel cuboid configuration reaches the best homogeneity without active shimming $\left(0.5 \mathrm{~B}_{\mathrm{s}}, 41 \mathrm{ppm}\right)$, while the Pseudo-Halbach configuration has the highest field strength $\left(0.9 \mathrm{~B}_{\mathrm{s}}, 994 \mathrm{ppm}\right)$, assuming perfect magnets. However, permanent magnet configurations suffer from imperfections, such as magnetisation, fabrication and positioning errors, which results in worse magnetic field homogeneities than expected from simulations using a fixed optimised parameter set. We present a sensitivity analysis for a magnetic cube and the results of studies of the variations in the magnetisation and angle of magnetisation of magnets purchased from different suppliers, composed of different materials and coatings, and of different sizes. We performed a detailed Monte Carlo simulation on the effect of the measured distribution of magnetic properties on the mentioned configurations. The cuboid design shows a mean homogeneity of $430 \mathrm{ppm}$ (std dev. $350 \mathrm{ppm}$ ), the Pseudo-Halbach has a mean homogeneity of 1086 ppm (std dev. 8 ppm).
\end{abstract}

Keywords-mobile NMR, magnet imperfections, permanent magnets, Halbach, field homogeneity, proof-reading-service

\section{INTRODUCTION}

Low-field and low-cost mobile microfluidic nuclear magnetic resonance (NMR) sensors are very suitable for applications in chemical process industry and in research, for example chemical analysis, biomedical applications, and flow measurements [1]-[9]. The design of a permanent magnet for an NMR sensor requires both a strong magnetic field and a high field homogeneity within a defined region of interest. In NMR, a high external magnetic field results in a high spectral resolution and detection sensitivity.

However, field inhomogeneities compromise the spectral resolution. Our aim with this research was to determine how the distribution of the properties of permanent magnets affect the magnetic field homogeneity of magnet configurations for mobile NMR devices.
In the literature, several magnet shapes for mobile NMR sensors have been reported. A broad overview of magnet developments up to 2009 can be found in Demas et al. [10]. U-shaped single-sided magnets [11], [12] and magnets with specially shaped iron pole magnets [13] have been used to explore surfaces. Mobile pseudo-Halbach configurations [14] and two cylindrical magnets [15] have been applied for solid and liquid NMR measurements. While the pseudo-Halbach generates a higher field, ranging from 0.7 to $2.0 \mathrm{~T}$ [16]-[18] compared to 0.35 to $0.6 \mathrm{~T}$ for the other configurations [5], [11]-[13], the reported field homogeneities without electric shimming seem to be independent of the design, ranging from $20 \mathrm{ppm}$ to $606 \mathrm{ppm} \mathrm{[1],} \mathrm{[5],} \mathrm{[15],} \mathrm{[18]-[20].} \mathrm{Comparing} \mathrm{the}$ two most reported mobile liquid NMR sensors, it further stands out that there is no obvious relation between the size of the sensor and the choice of the magnet configuration.

To achieve more insight into possible guidelines for the magnet design, in this paper a modelling study will be presented from which the homogeneity and field strength at specific locations in the gap of the magnet configuration is derived numerically. It is widely experienced that after building such a permanent magnet configuration, the homogeneity reached in practice does not exhibit the same results as in the simulation [16], [18], [21]-[23], which can be caused by several factors. The magnetisation of permanent magnets depends highly on the temperature, as well as on the remanent magnetisation [24]. This remanent magnetisation can change over time due to shock-induced demagnetisation [25], [26], external magnetic fields [27], a degrading of the magnetic material caused by oxidation [28], as well as broken or chipped off pieces (since magnets are very brittle) [21]. Next to material related differences, fabrication inaccuracies such as variations in the dimensions and magnetisation angles affect the field created by a permanent magnet. On top of that, magnet configurations can never be assembled perfectly. Errors in placement may induce a tilt or an axial offset of the magnet.

We carried out an extensive numerical sensitivity analysis of a single cubic magnet using these variations. We measured the variations in the magnetisation and magnetisation angle of magnets composed of different materials, with different coatings, and with different sizes, obtained from different manufacturers. The two main magnet configurations investigated are a system of two parallel magnets and a Pseudo-Halbach 


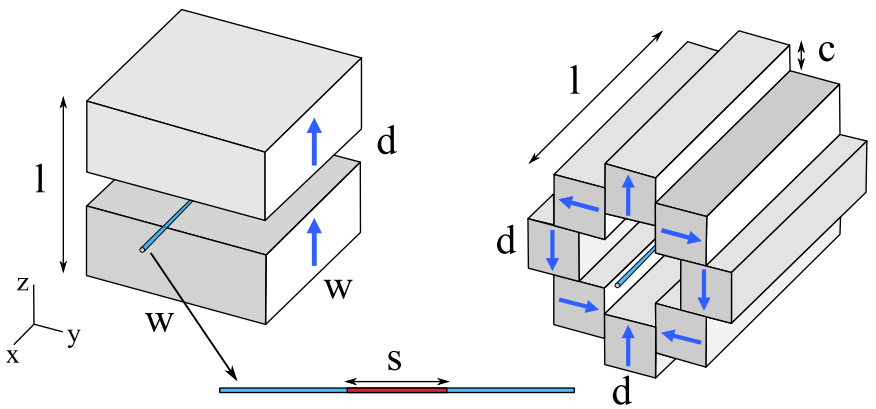

Fig. 1. Magnet configurations for microfluidic NMR. The sample under investigation is inside a tubular channel running through the center of the configurations, and has a length $s$. The arrows indicate the magnetisation of each individual magnet. Left: Cuboid configuration. The height of the stack $l$ is fixed at five times the gap between the magnets $d$. The width $w$ of the stack is optimised for minimum field inhomogeneity over the sample length $s$. Right: Pseudo-Halbach configuration. Again $l$ is fixed to $5 d$, but now the magnet recess $c$ is optimised.

configuration [10], shown in Fig. 1. One configuration of each type has been designed and optimised for the following boundary conditions. The sensitive length of the channel $(s)$ has been chosen to be the same as the gap size $(d)$. For example: In case a maximal magnet size of $50 \mathrm{~mm} \times 50 \mathrm{~mm} \times 50 \mathrm{~mm}$ is required, the gap size turns out to be $10 \mathrm{~mm}$. All dimension specifications are scalable and will be normalised by the gap length. Scaling the dimensions bigger or smaller will result in an increased or decreased sample length relative to the dimensions of the gap, while the magnetic field properties within the region of interest will stay the same. The magnetic field has been normalised to the residual magnetic flux density $B_{\mathrm{S}}(\mathrm{T})$ of the used magnetic material. The cuboid configuration consists of two cuboid magnets with a height of $2 d$ and a width of $4.72 d$. The Pseudo-Halbach configuration consists of eight bar magnets, each with the dimensions $d \times d \times 5 d$. The measured variations in the magnets have been used to perform a Monte Carlo simulation to provide insight into how the homogeneity of those configurations varies after assembling. The results have been verified with field measurements done with a Tesla meter. The sample channel in most published microfluidic NMR sensors has a high ratio of sample length over inner diameter $\left(s / d_{\mathrm{i}}\right)(5.0$ over $0.4 \mathrm{~mm}$ in [29], 30 over $1.0 \mathrm{~mm}$ in [30], and 2.9 over $0.15 \mathrm{~mm}$ in [31]). Therefore we focus on a high field homogeneity in mainly one dimension (x-axis).

\section{Methods}

\section{A. Determination of variation in magnet properties}

The variations in the properties of the magnets have been measured with a 3D Hall-probe (THM1176 Three-axis Hall Magnetometer, Metrolab). The setup for the configuration measurements contains a stable temperature environment $\left(38.0(5)^{\circ} \mathrm{C}\right)$ and a Hall sensor from Projekt Elektronik GmbH (Teslameter 3002/Transverse Probe T3-1,4-5,0-70) in combination with a motorised linear stage. Since the sensor is in a fixed position and only the magnet was moved for the measurement, field variations within the oven have no influence on the measurement. Different kinds of magnets have

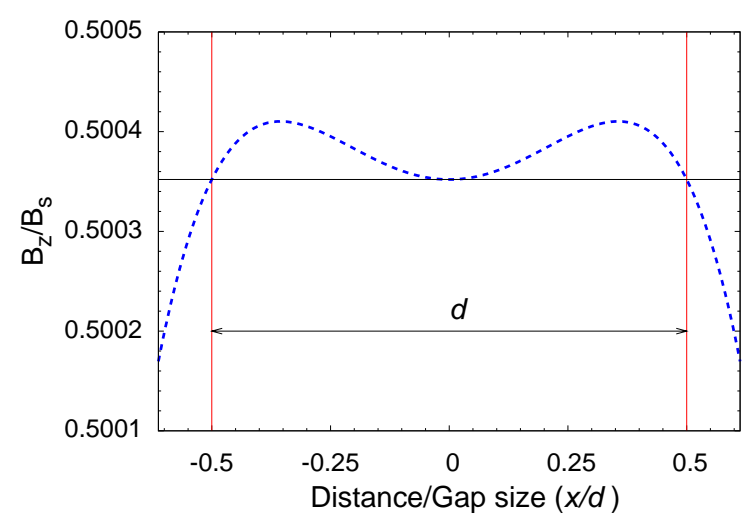

Fig. 2. Field profile of the cuboid configuration as a function of the relative distance from the centre between the magnets. In the optimised situation for a sample length equal to the gap size, the field in the centre equals the field at the edges $(x= \pm 0.5 d)$.

been purchased. We chose different materials, coatings, sizes and manufacturers, shown in Table I.

\section{B. Stray field calculation}

Calculations of the magnetic stray fields were performed using CADES simulation software, described by Delinchant et al. [32]. The magnetic interactions are modelled with the MacMMems tool, which uses the Coulombian equivalent charge method to generate a semi-analytic model.

$$
\mathbf{B}(\mathbf{r})=\iint \frac{\sigma\left(\mathbf{r}-\mathbf{r}^{\prime}\right)}{\left|\mathbf{r}-\mathbf{r}^{\prime}\right|^{3}} d s, \sigma=\mu_{0} \mathbf{M} \cdot \mathbf{n}
$$

Here, $\mathbf{B}$ is the magnetic field (T) and $\mathbf{M}$ the magnetisation of the permanent magnet $(\mathrm{A} / \mathrm{m}), \mathbf{r}$ and $\mathbf{r}^{\prime}$ define the observation point and its distance to the elementary field source area $d s$. The integral is taken over the surface of the magnets. $\sigma(\mathrm{T})$ is the magnetic surface charge, and $\mathbf{n}$ the unit vector normal to the surface.

The CADES framework, including a component generator, component calculator, and component optimiser, generated the final equations, which are used to calculate and optimise the designs.

\section{Design optimisation procedure}

The stray field calculations are used to optimize particular magnet configurations with respect to the inhomogeneity of the magnetic field over the length of the sample. This inhomogeniety is captured in a single valued metric defined as the root mean square of the difference between the $z$-component of the mean field $B_{\text {mean }}$ and the $z$-component of field along the sample $B_{z}$, averaged of the sample length $s$ and related to the mean field:

$$
\frac{1}{s B_{\text {mean }}} \int_{-s / 2}^{s / 2} \sqrt{\left(B_{z}-B_{\text {mean }}\right)^{2}} d x
$$

Minimisation of this metric leads to the simple rule that the field at the edges of the sample should equal the field 
TABLE I

PURCHASED PERMANENT MAGNETS.

\begin{tabular}{|c|c|c|c|c|c|c|}
\hline Manufacturer & $\begin{array}{l}\text { Dimension } \\
(\mathrm{mm})\end{array}$ & Material & $\begin{array}{l}B_{\mathrm{r}} \\
(\mathrm{mT})\end{array}$ & $\begin{array}{l}B H_{\max } \\
\left(\mathrm{kJ} / \mathrm{m}^{3}\right)\end{array}$ & Coating & Abbreviation \\
\hline Supermagnete & $45 \times 30^{1}$ & $\mathrm{NdFeB}(\mathrm{N} 45)$ & $1320-1360$ & $340-372$ & $\mathrm{Ni}-\mathrm{Cu}-\mathrm{Ni}$ & Su45Nd45NCN \\
\hline Supermagnete & $7 \times 7 \times 7$ & $\mathrm{NdFeB}(\mathrm{N} 42)$ & $1290-1330$ & $318-342$ & $\mathrm{Ni}-\mathrm{Cu}-\mathrm{Ni}$ & Su7Nd42NCN \\
\hline Supermagnete & $7 \times 7 \times 7$ & $\mathrm{NdFeB}(\mathrm{N} 42)$ & $1290-1330$ & $318-342$ & $\mathrm{Ni}-\mathrm{Cu}$ & Su7Nd42NC \\
\hline HKCM & $7 \times 7 \times 7$ & $\mathrm{NdFeB}(\mathrm{N} 35)$ & $1180-1230$ & $263-287$ & $\mathrm{Ni}$ & HK7Nd35N \\
\hline HKCM & $7 \times 7 \times 7$ & Sm2Co17 (YXG28) & $1030-1080$ & $207-220$ & $\mathrm{Ni}$ & HK7Sm28N \\
\hline Schallenkammer Magnetsyteme & $7 \times 7 \times 7$ & Sm2Co17 (YXG26H) & $1020-1050$ & $191-207$ & - & Sc7Sm26 \\
\hline
\end{tabular}

${ }^{1}$ diameter $\times$ height, axially magnetised

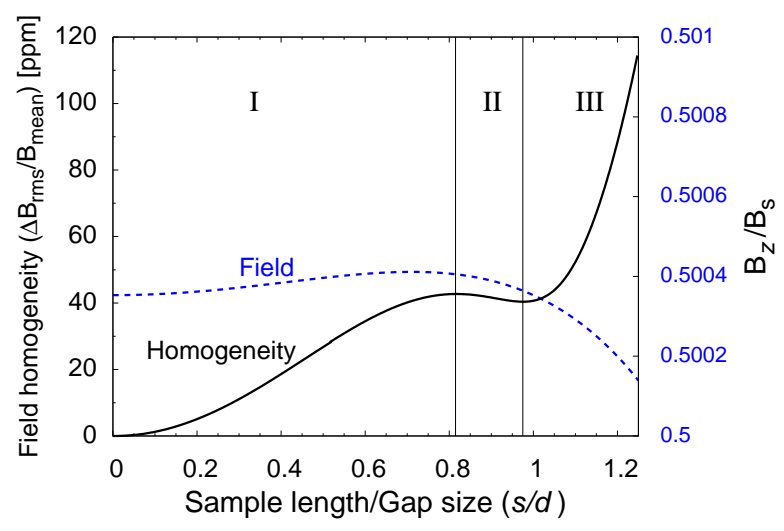

Fig. 3. Field inhomogeneity and field as a function of the sample length for the cuboid configuration. The homogeneity has been optimised for a sample length equal to the gap size. With increasing sample length, both the field and the field inhomogeneity increase theoretically (region I). The field reaches a local maximum of $0.50041 B_{\mathrm{s}}$ at a distance of $0.71 \mathrm{~d}$ from the centre. Above this distance the homogeneity of the sample stays approximately the same (region II). When the sample length increases more than the gap size, the inhomogeneity strongly increases (region III). If we choose the field at the edge of the sample to be equal to the centre of the sample, we are very close to the minimum homogeneity in region II.

in the center. We illustrate this for the cuboid configuration, illustrated in Fig. 1. Fig. 2 shows the magnetic field along the sample of the optimised cuboid configuration, in which the field is the same in the centre and at the edge of a sample. The field is symmetric, showing a valley in the middle and two peaks in the directions of the edges. After those maxima, the field decreases with the distance to the centre.

Fig. 3 shows how the field homogeneity develops with increasing sample length while keeping the previously optimised parameter set constant. Three regions can be seen. In the first one the field increases from $0.50035 B_{\mathrm{s}}$ to $0.50041 B_{\mathrm{s}}$, which means that the minimum field of $0.50035 B_{\mathrm{s}}$ stays the same while the maximum field is increasing until it reaches its global maximum, hence the inhomogeneity is also increasing. In the second region the inhomogeneity stays almost constant. In the third region the field decreases below the previous minimum, which results in a drastic increase of the inhomogeneity. Therefore, the lowest inhomogeneity between two points can either be reached by keeping the sample as short as possible or when the field at the sample edges is approximately equal to the field at the center. Since the signal in NMR is proportional to the sample volume, we optimise for the latter condition.
TABLE II

MEASURED COEFFICIENT OF VARIATION (CV) OF RESIDUAL MAGNETIC FLUX DENSITY AND STANDARD DEVIATIONS OF MAGNETISATION ANGLE OF MAGNETS WITH DIFFERENT MATERIALS, COATINGS, SIZES AND MANUFACTURERS.

\begin{tabular}{lcc}
\hline \hline Magnet & $B_{\text {mean }}, C V[\%]$ & $\phi\left[^{\circ}\right]$ \\
\hline Su45Nd45NCN & $0.7(3)$ & $0.0(1)^{1}$ \\
Su7Nd42NCN & $0.8(2)$ & $0.7(2)$ \\
Su7Nd42NC & $0.6(3)$ & $0.0(1)$ \\
HK7Nd35N & $0.3(3)$ & $0.4(2)$ \\
HK7Sm28N & $1.0(3)$ & $0.2(1)$ \\
Sc7Sm26 & $1.6(2)$ & $1.0(2)$ \\
\hline \hline & The values between brackets are the absolute standard \\
\multicolumn{2}{l}{ Thrors of the last shown digits. }
\end{tabular}

\section{RESULTS AND DISCUSSION}

The field uniformity of the various designs is determined by the design itself as well as the manufacturability. One major point of concern is the variation in the value and alignment of the magnetic moment of the permanent magnets. Therefore we first present the distribution of these properties for a range of commercial magnets. We subsequently optimise the designs with respect to uniformity and analyse their sensitivity to magnet variation using sensitivity matrices and Monte-Carlo simulations. These model predictions are than compared with six realisations of the different designs.

\section{A. Variation of properties of commercial permanent magnets}

We measured the variations in the magnetisation and magnetisation angle of magnets obtained from different companies (Supermagnete, HKCM and Schallenkammer Magnetsysteme), compositions ( $\mathrm{NdFeB} \mathrm{N45,} \mathrm{NdFeB} \mathrm{N42,} \mathrm{Sm2Co17}$ YXG28, Sm2Co17 YXG26H), coatings (Ni-Cu-Ni, Ni-Cu, $\mathrm{Ni}$, no coating), and sizes (cylinders with a diameter of 45 and height of $30 \mathrm{~mm}$ or cubes of $7 \mathrm{~mm} \times 7 \mathrm{~mm} \times 7 \mathrm{~mm}$ ). Of each set, 50 magnets were analysed. An overview of the distributions in residual magnetic flux density and angle of magnetisation is given in Table II. The raw data is provided in the supplementary material (Appendix A).

On average, the residual flux density varies by $1 \%$ of $B_{\text {mean }}$. The cylindrical magnet, which has a more than 50 times higher magnetic volume than the cubes, shows roughly the same variation in magnetisation. From this, we can conclude that inaccuracies in the dimensions are not the main cause of the variation in the magnetisation. The uncoated $\mathrm{Sm} 2 \mathrm{Co} 17$ shows a higher variation in magnetisation than the coated magnets, 


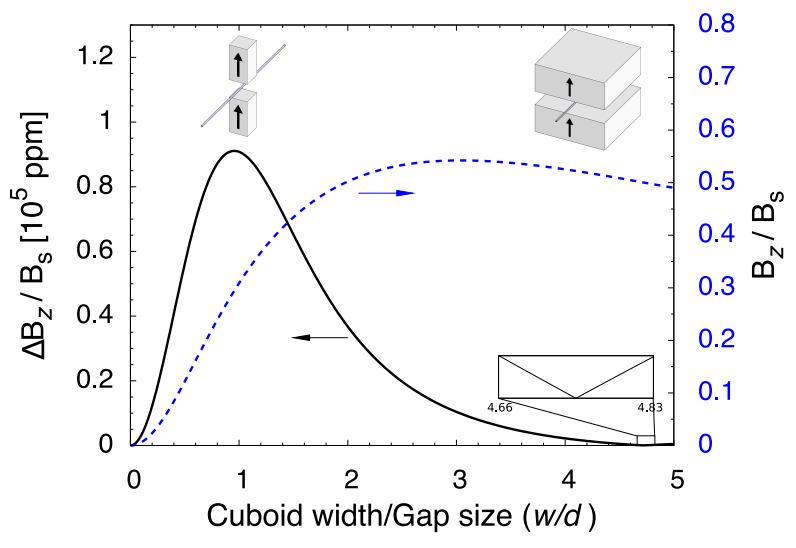

Fig. 4. Field strength in the middle of the configuration and difference of the field centre and the edge of the sample, both as functions of the ratio of the cuboid width over the length. The field increases up to $0.54 B_{\mathrm{S}}$ at a cuboid width of 3.0375 times the gap size. The inset shows the field difference dropping to zero at a width/gap ratio of 4.72 .

which could be caused by oxidation or small damage to the magnet since unprotected sharp edges of magnets tend to break off easily. Different coatings do not show a clear trend regarding the magnetisation standard variation or the variation in the magnetisation angle. The offset angle varies on average by less than $1^{\circ}$. There is no clear relation between the variation in magnetisation strength or orientation and material, coating or manufacturer.

\section{B. Design optimisation}

The optimisation method described in section II-C was applied to both the cuboid and the pseudo-Halbach design.

a) Optimisation of the cuboid configuration: The cuboid configuration consists of two parallel cuboid magnets. The length $L$ of the entire configuration has been chosen to be five times the gap size $d$. The width $W$ was used to tune the field in between the magnets. The optimisation procedure aims to find a width for which the field in the centre and at the sample edge is the same.

Fig. 4 shows that the magnetic field in the centre increases to its maximum of $0.54 B_{\mathrm{S}}$ at a width of $3.0375 d$. Increasing the width further results in a reduction of the magnetic field, caused by the larger distance from the edges of the magnet to the centre. The difference between the magnetic field in the centre and that at the sample edge increases until it reaches a maximum, when the width equals the gap size. From this point the difference decreases until it reaches a minimum at a width/gap ratio of 4.72 . The stray field at a distance equal to the gap size is $0.24 B_{\mathrm{s}}$.

b) Pseudo-Halbach: The pseudo-Halbach configuration consists of eight magnets, arranged in such a way that the field in the bore is enhanced while the external stray field is minimised. The magnets have a fixed dimension $d \times d \times 5 d$. To tune the homogeneity, the position of the magnets in the corners is fixed, while the other magnets are spread out over a distance $c$ (Fig. 1). The width starts at $w=3 d$ to ensure a minimum bore width $d$ and ends at $w=l$, due to the previously chosen boundary conditions.

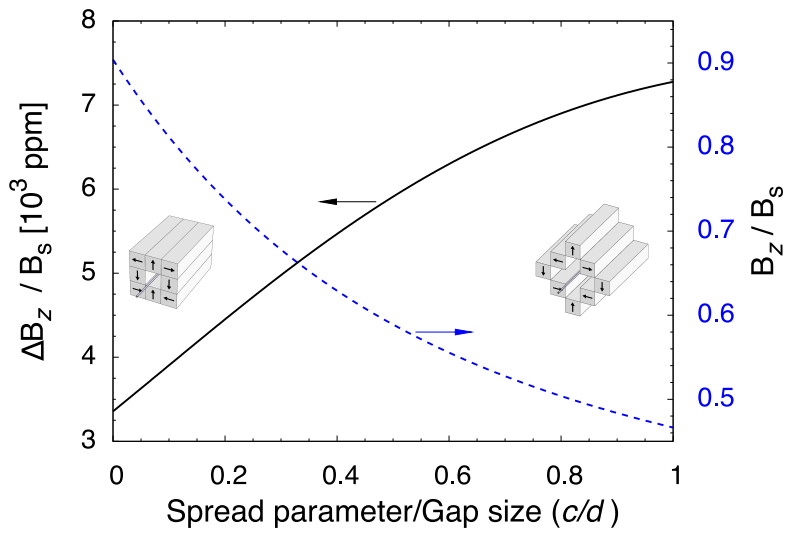

Fig. 5. Spreading the middle magnets has been used to change the normalised field strength and field difference. At a spread parameter $c=0$, a minimal field difference of $3365 \mathrm{ppm}$ and a field strength of $0.90 B_{\mathrm{s}}$ can be reached.

TABLE III

COMPARISON OF MAGNETIC PROPERTIES OF DIFFERENT MAGNET CONFIGURATIONS.

\begin{tabular}{lccc}
\hline \hline & $\begin{array}{c}B_{\max } \\
{\left[B_{\mathrm{s}}\right]}\end{array}$ & $\begin{array}{c}B_{\text {stray }} \\
{\left[B_{\mathrm{s}}\right]}\end{array}$ & $\begin{array}{c}\Delta B_{\text {rms }} / B_{\text {mean }} \\
{[\mathrm{ppm}]}\end{array}$ \\
\hline Cuboid & 0.5 & 0.24 & 41 \\
Pseudo-Halbach & 0.9 & 0.07 & 994 \\
\hline \hline
\end{tabular}

Spreading the configuration increases the distance of the middle magnets, which produces a decreased magnetic field strength (Fig. 5). With this configuration the convex field profile has no chance to change to a concave profile. Therefore a minimum can not be reached. With the most compact magnet arrangement $(c=0)$, a field of $0.9 B_{\mathrm{s}}$ and a field difference of $3365 \mathrm{ppm}$ can be achieved. The stray field at a distance equal to the gap size from the surface is $0.07 B_{\mathrm{s}}$.

In table III the major specifications of the two optimised configurations are compared. The pseudo-Halbach configuration achieves $0.9 B_{\mathrm{s}}$, a 1.8 times higher field than the Cuboid configuration, while the stray field at a distance of $d$ from the magnet surface is $0.07 B_{\mathrm{s}}$, which is 3.4 times lower. In terms of homogeneity, the Cuboid configuration achieves a homogeneity of $41 \mathrm{ppm}$, which, compared to the pseudoHalbach configuration, is 24.2 times better.

Neither of the two designs reach a field uniformity below $0.01 \mathrm{ppm}$, which is required for high resolution NMR, so additional field shimming will remain necessary. However, it is interesting to analyse whether high resolution NMR systems without shimming are reachable by reducing the sample length. Therefore, we optimised the homogeneity of the configuration as a function of sample lengths, while keeping the outer boundary conditions intact. Fig. 6 shows how the homogeneity improves with a reducing ratio of the gap size to the sample length. The cuboid configuration can indeed reach in theory $0.01 \mathrm{ppm}$ with a sample length of $0.22 d$. The pseudoHalbach configuration however needs an absurd sample length of $0.01 d$ to reach the critical value. 


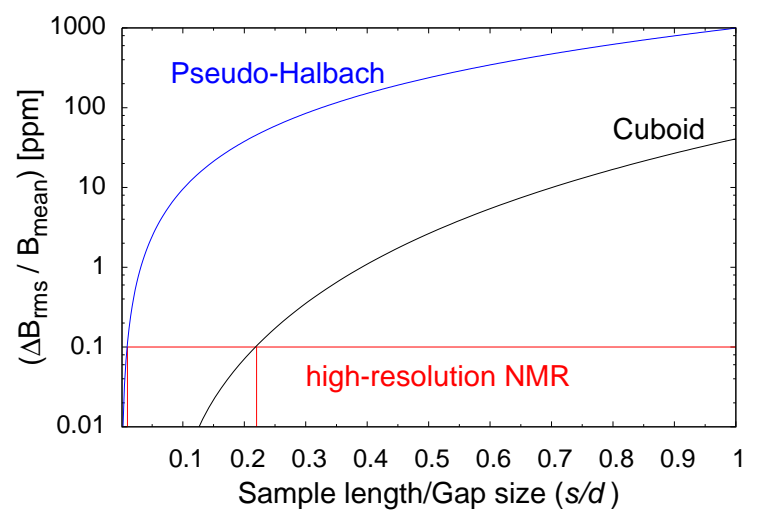

Fig. 6. Inhomogeneity of the magnetic field as a function of the sample length/gap ratio with a constant configuration width/gap ratio of 5 . For every sample length, the width of the cuboid configurations has been optimised to reach the lowest possible inhomogeneity. For a homogeneity reasonable for NMR applications of $0.1 \mathrm{ppm}$, the sample length for a cuboid configuration needs to be $0.22 d$, whereas it has to become unrealistically short $(0.01 d)$ for the pseudo-Halbach configuration.

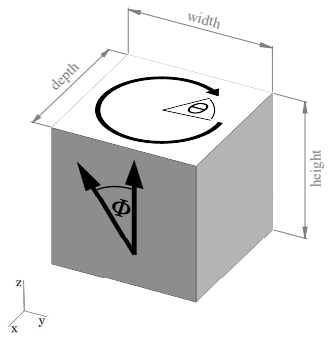

Fig. 7. Schematic drawing of the cubic magnet used for sensitivity analysis. The length width and depth have a nominal size of $a$. The arrows indicate the direction of magnetisation. $\phi$ shows the total offset angle, $\theta$ the offset direction in cylindrical coordinates.

\section{Influence of variations in the magnet properties}

To analyse the effect of variation in magnet properties and positioning on the performance of both designs, we applied a two step approach. First we analysed the sensitivity of the magnetic field to the variation in strength and position of a single cubic magnet using the method of sensitivity matrices. From this we determined that variations in magnetic moment and angle of magnetisation are most severe. Focussing on these two parameters only, we analysed the combined effect of all magnets using a Monte-Carlo approach.

1) Single cubic magnet: We determined the sensitivity of the magnetic stray field of a single cubic magnet of nominal size $a$ to a variation of the dimensions, position, and tilt of the magnet, as well as in the magnetisation strength and angle (Fig. 7). We consider the field components $B_{x}, B_{y}, B_{z}$ at a point above the centre of the top (north) face, at a height of $10 \%$ of the length $a$ of the edge of the magnet.

Table IV shows the most important elements of the sensitivity matrix of the magnetic field in the $x$ and $z$-directions on the $x$-axis at a distance of $0.1 a$, given as percentages of $B_{\mathrm{s}}$. Parameters related to the sizes have been varied by $10 \%$ of the length of the edge of the cube. Parameters related to the angle have been varied by $1^{\circ}$. Appendix $\mathrm{B}$ gives the sensitivities for
TABLE IV

SENSITIVITY MATRIX OF THE MAGNETIC FIELD COMPONENTS ( $B_{x}$ AND $B_{z}$ ) AT A DISTANCE OF $0.1 a$ ABOVE THE CENTER OF A CUBIC MAGNET WITH THE EDGE LENGTH $a$.

\begin{tabular}{lcrr}
\hline \hline & Variation & \multicolumn{1}{c}{$B_{x}(\%)$} & \multicolumn{1}{c}{$B_{z}(\%)$} \\
\hline$M$ & $1 \%$ & 0.00 & 1.00 \\
tilt $x$ & $1^{\circ}$ & 0.00 & 0.00 \\
tilt $y$ & $1^{\circ}$ & 0.61 & 0.00 \\
$\phi\left(\theta=0^{\circ}\right)$ & $1^{\circ}$ & 0.00 & 0.00 \\
$\phi\left(\theta=90^{\circ}\right)$ & $1^{\circ}$ & -0.87 & 0.00 \\
$x$ & $0.1 a$ & -1.09 & 0.00 \\
$z$ & $0.1 a$ & 0.00 & -2.17 \\
\hline \hline
\end{tabular}

$B_{y}$ and the field at $x=0.1 a$ in Table IX, as well as the absolute field values in Figure 13.

The first row in Table IV shows that the $B_{\mathrm{Z}}$ component changes proportionally with the magnetisation. Since the $B_{\mathrm{X}}$ component is zero (see Figure 13 of appendix B ), variation in magnetisation has no effect. Similarly, a tilt of the cube or rotation of the magnetisation around the $y$-axis has a significant influence only on $B_{\mathrm{x}}$, but not on $B_{\mathrm{z}}$. Displacement of the cube has an effect only on the field components in the direction of displacement. The effect is relatively small: a $10 \%$ variation in position only lead to a $2 \%$ variation in field strength.

2) Monte-Carlo simulations: To analyse the combined effect of all magnets on the field, we performed a Monte Carlo simulation with 50000 draws. Based on the above analysis of the cube, we consider only variation in the magnetisation strength and direction. Since for the two configurations the dimensional variation is smaller than $0.03 \mathrm{a}$, no dimensional errors were considered. Normal distributions were assumed, with standard deviations of $1^{\circ}$ and $1 \%$ for strength and angle respectively.

Fig. 8 shows the distribution and probability plot of the simulated homogeneities of the magnetic field in the $z$ direction. The mean homogeneity of the cuboid configuration is $430 \mathrm{ppm}$, the pseudo-Halbach configuration achieves $1086 \mathrm{ppm}$. However, the cuboid configuration has a high spread in the homogeneity (standard deviation $350 \mathrm{ppm}$ ) while the pseudo-Halbach has a standard deviation of only $8 \mathrm{ppm}$. With a probability of $94.4 \%$, both the cuboid configuration and the pseudo-Halbach configuration obtain a homogeneity of $1098 \mathrm{ppm}$ or better. With a probability of $10 \%$, the cuboid configuration achieves $64 \mathrm{ppm}$ whereas the pseudo-Halbach achieves not less than $1076 \mathrm{ppm}$.

The reason for the strong sensitivity of the cuboid configuration to magnet variations is largely due to the distribution in magnetisation direction. Table $\mathrm{V}$ ) shows the sensitivity of the $z$ component of the field at the center and the edge of the sample to a variation of $1^{\circ}$ of the magnetisation $(x=d / 2)$ angle. At the edge of the sample, the cuboid configuration is ten times more sensitive to angular variations.

\section{Verification of simulations with implementations}

Both configurations were assembled and measured three times. The measurement results are shown in Table VI. There is a small spread in the homogeneity of the pseudo-Halbach (mean value of $1032 \mathrm{ppm}$ and standard deviation of $90 \mathrm{ppm}$ ). 

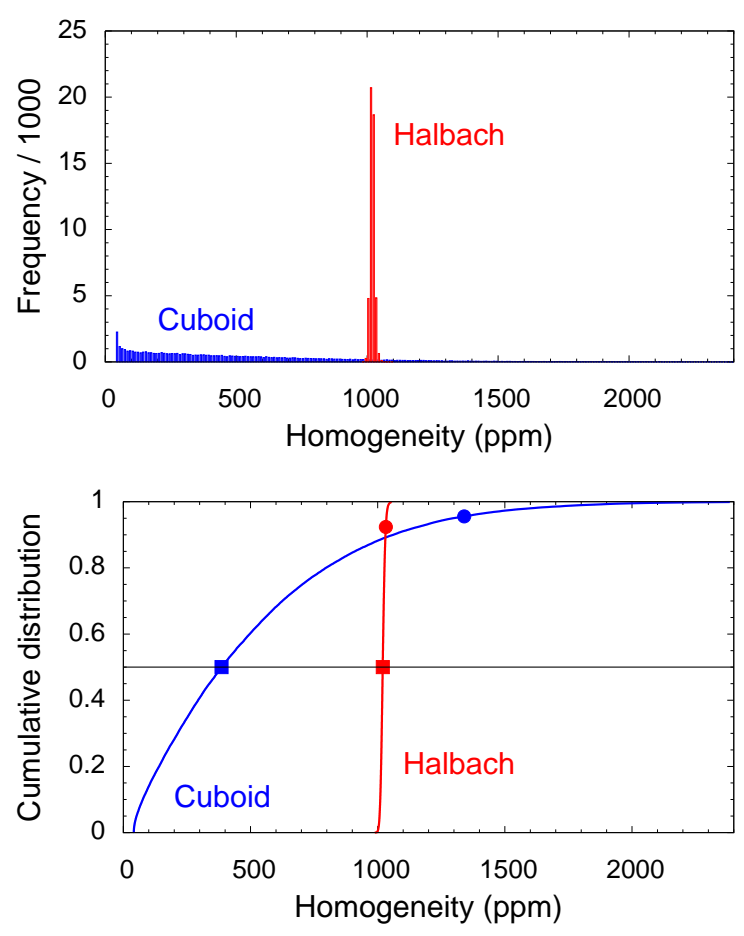

Fig. 8. Density plot (top) and cumulative distribution function (bottom) of the Monte Carlo simulation for the cubic and Pseudo-Halbach configurations. The simulation shows that the Pseudo-Halbach configuration has a mean homogeneity of $1020 \mathrm{ppm}$, while the cuboid configuration reaches $386 \mathrm{ppm}$ (Squares in bottom figure). The circles indicate the average inhomogeneity measured on three implementations of each configuration (Table VI).

TABLE V

SENSITIVITY TO VARIATION IN MAGNETISATION ANGLE OF $B_{z}$ AT THE CENTER AND EDGE OF THE SAMPLE FOR THE PSEUDO-HALBACH AND CUBOID CONFIGURATION

\begin{tabular}{lccl}
\hline \hline & $B_{z}(x=0)$ & $B_{z}(x=d / 2)$ & \\
\hline Halbach - top/bottom & 0 & 59 & $\mathrm{ppm} / \mathrm{deg}$ \\
Halbach - side & 0 & 0 & $\mathrm{ppm} / \mathrm{deg}$ \\
Halbach - corner & 0 & 81 & $\mathrm{ppm} / \mathrm{deg}$ \\
Cuboid & 0 & 985 & $\mathrm{ppm} / \mathrm{deg}$ \\
\hline \hline
\end{tabular}

A larger variation was found for the cuboid configuration (1340 and $800 \mathrm{ppm})$. (Raw data of the six systems is listed in Table XIII of appendix C ).

The three implementations represent a draw from the 50000 Monte Carlo simulations shown in figure Fig. 8. From these curves we can estimate that the chance to realise a cuboid configuration with an inhomogeneity as bad as $1340 \mathrm{ppm}$ is in the order of $5 \%$ (blue dot in figure). Similarly, a pseudoHalbach configuration with a inhomogeneity of $1032 \mathrm{ppm}$ or worse has a chance of $8 \%$ of occurring. These likelihoods are low, but not unrealistic. More implementations would be required to determine whether other variation than magnetisation strength and direction should be considered.

In general, the pseudo-Halbach configuration has a more predictable field profile, which makes this design more favourable for industrial applications than the cuboid configuration. Since shimming is needed anyway, a measurement of the field profile is not necessary. We therefore recommend
TABLE VI

MEAN (STANDARD DEVIATION) OF THE HOMOGENEITY OF THE FIELD IN THE $z$-DIRECTION OF MEASURED AND SIMULATED MAGNET CONFIGURATIONS

\begin{tabular}{lcc}
\hline \hline & $\begin{array}{c}\text { Measured } \\
\text { Inhomogeneity } \\
{[\mathrm{ppm}]}\end{array}$ & $\begin{array}{c}\text { Simulated } \\
\text { Inhomogeneity } \\
{[\mathrm{ppm}]}\end{array}$ \\
\hline Cuboid & $1340(800)$ & 386 \\
Pseudo-Halbach & $1032(90)$ & 1020 \\
\hline \hline
\end{tabular}

restricting the use of the cuboid configurations to research systems, where selecting the magnets and measuring the final assembly is feasible.

\section{CONCLUSION}

We have investigated the effect on the homogeneity of the field of permanent magnet configurations for mobile NMR applications of variations in the properties of the magnets. We measured the variations in the magnetisation and magnetisation angle of permanent magnets but could not observe a decisive difference between the manufacturers, materials, or magnet coatings. On average, the standard deviation of the magnetisation is less than $1 \%$ and for the variations in the magnetisation angle it is less than $1^{\circ}$.

We compared a cuboid and a pseudo-Halbach magnet configuration, in terms of their field strength and field homogeneity, for our optimised boundary conditions, in which the sample length $s$ is equal to the gap size $d$ and the whole configuration should fit in a box with an edge length five times the gap size. For a fixed parameter set, assuming perfectly magnetised magnets, the field in the centre of the cuboid configuration is $0.5 B_{\mathrm{s}}$ and its homogeneity is $41 \mathrm{ppm}$. For the same boundary conditions, the pseudo-Halbach configuration achieves a higher field $\left(0.9 B_{\mathrm{S}}\right)$ in the centre but less homogeneity $(994 \mathrm{ppm})$. It is worth mentioning that the pseudo-Halbach configuration has a much lower stray field, and so less interference with the environment, than the cuboid configuration.

For samples with a length the same as the gap size, the theoretical homogeneity of both configurations is above the sub-ppm range, which is necessary to produce a high resolution spectrum. Optimising the homogeneity for shorter samples while respecting the maximum outer dimensions yields in a much better homogeneity. Using a sample length of $0.22 d$ improves the homogeneity from 41 to $0.1 \mathrm{ppm}$ for the cuboid configuration, whereas the pseudo-Halbach configuration would need a impractical sample length of $0.01 \mathrm{~d}$.

We analysed the effect of the variation in magnetic properties on the uniformity of the generated fields. The sensitivity matrix shows that the magnetisation, magnetisation angle, and tilt have the most significant influence on the magnetic field. Positioning errors mainly change the field, in case the positioning variation is in the same direction as the field. Theoretically, the cuboid has good homogeneity (on average $430 \mathrm{ppm}$ ), but the effect of variation in the magnets' properties is large (standard deviation $350 \mathrm{ppm}$ ). The pseudo-Halbach configuration has worse homogeneity $(1080 \mathrm{ppm})$, but is 44 times less sensitive to variation in the properties of the magnet. 
We verified the modelled field inhomogeneities with three realisations for each of the two designs. The average inhomogeneity agree within measurement error with the model.

Based on our analysis, we advise using the cuboid configuration for scientific use, where it is possible to preselect the permanent magnets and the external stray field is not a big issue. Mechanical shimming of this configuration can be done, changing the distance between the magnets (counteracting magnetisation differences) or by tilting the magnet (counteracting magnetisation angle variations). Using rather large magnets helps to achieve the homogeneity needed for NMR measurements. If preselecting the magnets is not an option, we recommend the pseudo-Halbach configuration, which has a more robust homogeneity regarding variations in the magnetisation and angle. The field profile of this configuration is predictable, which makes it easier to shim afterwards to achieve the field homogeneity needed for NMR applications. Also the lower stray field makes this configuration easier to handle and therefore more favourable especially for industrial applications.

\section{ACKNOWLEDGEMENTS}

This work is part of the research programme FLOW+ with project number 15025 , which is (partly) financed by the Dutch Research Council (NWO). The authors thank Jankees Hogendoorn and Lucas Cerioni of Krohne New Technologies BV for their input and support.

\section{REFERENCES}

[1] M. Meribout and S. Sonowan, "Optimal halbach magnet array design for portable NMR targeting multiphase flow metering applications," IEEE Transactions on Magnetics, vol. 55, no. 1, pp. 1-7, jan 2019.

[2] S. S. Zalesskiy, E. Danieli, B. Blümich, and V. P. Ananikov, "Miniaturization of NMR systems: Desktop spectrometers, microcoil spectroscopy, and "NMR on a chip" for chemistry, biochemistry, and industry," Chemical Reviews, vol. 114, no. 11, pp. 5641-5694, apr 2014.

[3] J. Mitchell, L. Gladden, T. Chandrasekera, and E. Fordham, "Low-field permanent magnets for industrial process and quality control," Progress in Nuclear Magnetic Resonance Spectroscopy, vol. 76, pp. 1-60, jan 2014.

[4] E. Danieli, J. Perlo, B. Blümich, and F. Casanova, "Small magnets for portable NMR spectrometers," Angewandte Chemie International Edition, vol. 49, no. 24, pp. 4133-4135, may 2010.

[5] H. Lee, E. Sun, D. Ham, and R. Weissleder, "Chip-NMR biosensor for detection and molecular analysis of cells," Nature Medicine, vol. 14, no. 8, pp. 869-874, jul 2008

[6] D. Kreyenschulte, E. Paciok, L. Regestein, B. Blümich, and J. Büchs, "Online monitoring of fermentation processes via non-invasive low-field NMR," Biotechnology and Bioengineering, vol. 112, no. 9, pp. 18101821, may 2015.

[7] M. K. Sørensen, O. Jensen, O. N. Bakharev, T. Nyord, and N. C. Nielsen, "NPK NMR sensor: Online monitoring of nitrogen, phosphorus, and potassium in animal slurry," Analytical Chemistry, vol. 87, no. 13, pp. 6446-6450, jun 2015.

[8] M. K. Sørensen, M. S. Vinding, O. N. Bakharev, T. Nesgaard, O. Jensen, and N. C. Nielsen, "NMR sensor for onboard ship detection of catalytic fines in marine fuel oils," Analytical Chemistry, vol. 86, no. 15, pp. 7205-7208, jul 2014.

[9] G. Mozzhukhin, G. Kupriyanova, S. Mamadazizov, A. Maraşlı, and B. Rameev, "Low-field 14n nuclear magnetic resonance for detection of dangerous liquids," Chemical Physics, vol. 513, pp. 129-134, sep 2018.

[10] V. Demas and P. J. Prado, "Compact magnets for magnetic resonance," Concepts in Magnetic Resonance Part A, vol. 34A, no. 1, pp. 48-59, jan 2009.
[11] B. Blümich, P. Blümler, G. Eidmann, A. Guthausen, R. Haken, U. Schmitz, K. Saito, and G. Zimmer, "The NMR-mouse: construction, excitation, and applications," Magnetic Resonance Imaging, vol. 16, no. 5-6, pp. 479-484, jun 1998.

[12] W. Meethan, I. Thomas, and C. Thomas, "Calculation of the sensitive region of a u-shaped permanent magnet for a single-sided NMR spectrometer," Chiang Mai University Journal of Natural Sciences, vol. 13, no. $1,2014$.

[13] A. E. Marble, I. V. Mastikhin, B. G. Colpitts, and B. J. Balcom, "An analytical methodology for magnetic field control in unilateral NMR," Journal of Magnetic Resonance, vol. 174, no. 1, pp. 78-87, may 2005.

[14] M. W. Vogel, A. Giorni, V. Vegh, R. Pellicer-Guridi, and D. C. Reutens, "Rotatable small permanent magnet array for ultra-low field nuclear magnetic resonance instrumentation: A concept study," PLOS ONE, vol. 11, no. 6, p. e0157040, jun 2016.

[15] N. Sun, Y. Liu, L. Qin, H. Lee, R. Weissleder, and D. Ham, "Small NMR biomolecular sensors," Solid-State Electronics, vol. 84, pp. 13 21, 2013, selected Papers from the ESSDERC 2012 Conference.

[16] E. Danieli, J. Perlo, B. Blümich, and F. Casanova, "Small magnets for portable NMR spectrometers," Angewandte Chemie International Edition, vol. 49, no. 24, pp. 4133-4135, may 2010.

[17] M. C. Tayler and D. Sakellariou, "Low-cost, pseudo-halbach dipole magnets for NMR," Journal of Magnetic Resonance, vol. 277, pp. 143148, apr 2017.

[18] G. Moresi and R. Magin, "Miniature permanent magnet for table-top NMR," Concepts in Magnetic Resonance, vol. 19B, no. 1, pp. 35-43, 2003.

[19] R. S. Sahebjavaher, K. Walus, and B. Stoeber, "Permanent magnet desktop magnetic resonance imaging system with microfabricated multiturn gradient coils for microflow imaging in capillary tubes," Review of Scientific Instruments, vol. 81, no. 2, p. 023706, feb 2010.

[20] J. Chen and C. Xu, "Design and analysis of the novel test tlibe magnet as a device for portable nuclear magnetic resonance," IEEE Transactions on Magnetics, vol. 43, no. 9, pp. 3555-3557, 2007, cited By 4.

[21] J. Horton, J. Wright, and J. Herchenroeder, "Fracture toughness of commercial magnets," IEEE Transactions on Magnetics, vol. 32, no. 5, pp. 4374-4376, 1996

[22] H. Soltner and P. Blümler, "Dipolar halbach magnet stacks made from identically shaped permanent magnets for magnetic resonance," Concepts in Magnetic Resonance Part A, vol. 36A, no. 4, pp. 211-222, jul 2010.

[23] A. Ambrisi, A. Formisano, and R. Martone, "Tolerance analysis of nmr magnets," IEEE Transactions on Magnetics, vol. 46, no. 8, pp. 27472750, 2010, cited By 9.

[24] M. Haavisto, H. Kankaanpaa, and M. Paju, "Estimation of timedependent polarization losses in sintered ndfeb permanent magnets," IEEE Transactions on Magnetics, vol. 47, no. 1, pp. 170-174, jan 2011.

[25] Y.-F. Li, M.-G. Zhu, W. Li, D. Zhou, F. Lu, L. Chen, J.-Y. Wu, Y. Qi, and A. Du, "The impact induced demagnetization mechanism in ndfeb permanent magnets," Chinese Physics Letters, vol. 30, no. 9, p. 097501, sep 2013.

[26] E. B. Royce, "Anomalous shock-induced demagnetization of nickel ferrite," Journal of Applied Physics, vol. 37, no. 11, pp. 4066-4070, oct 1966.

[27] C. J. Lee, C. I. Lee, and G. H. Jang, "Source and reduction of uneven magnetization of the permanent magnet of a HDD spindle motor," IEEE Transactions on Magnetics, vol. 47, no. 7, pp. 1929-1932, jul 2011.

[28] Y. Li, H. Evans, I. Harris, and I. Jones, "The oxidation of ndfeb magnets," Oxidation of Metals, vol. 59, no. 1/2, pp. 167-182, 2003.

[29] J. Gardeniers, J. Bart, A. Kolkman, A.-J. de Vries, J. Janssen, P. van Bentum, K. Ampt, S. Wijmenga, and A. Kentgens, "Microfluidic highresolution NMR chip for biological fluids," in TRANSDUCERS 2009 - 2009 International Solid-State Sensors, Actuators and Microsystems Conference. IEEE, jun 2009.

[30] A. Kalfe, A. Telfah, J. Lambert, and R. Hergenröder, "Looking into living cell systems: Planar waveguide microfluidic NMR detector for in vitro metabolomics of tumor spheroids," Analytical Chemistry, vol. 87, no. 14, pp. 7402-7410, jul 2015.

[31] E. E. McDonnell, S. Han, C. Hilty, K. L. Pierce, and A. Pines, "Nmr analysis on microfluidic devices by remote detection," Anal. Chem., vol. 77, pp. 8109-8114, 2005.

[32] B. Delinchant, D. Duret, L. Estrabaut, L. Gerbaud, H. N. Huu, B. D. Peloux, H. Rakotoarison, F. Verdiere, and F. Wurtz, "An optimizer using the software component paradigm for the optimization of engineering systems," COMPEL - The international journal for computation and mathematics in electrical and electronic engineering, vol. 26, no. 2, pp. 368-379, 2007. 


\section{APPENDIX}

\section{APPENDIX A}

\section{DISTRIBUTION OF PERMANENT MAGNET PROPERTIES}

We measured the magnetisation strength and orientation for a range of commercially available permanent magnets (listed in table I of main text). The results are summarized in table II in the main text. The underlying data is reported in this appendix.

Figure 9 shows the cumulative distribution of the residual flux density, normalized to the mean value. The measurement uncertainty is estimated from the cumulative distribution for 50 measurements of the same magnet (black curve). The distribution in magnetisation of the commercial magnets exceeds our measurement uncertainty. The standard deviation is in the order of $1 \%$.

In a similar fashion, the variation in field direction was measured (Figure 10). The base uncertainly measurement is again shown as a black curve. The histogram presentation is shown in figure 11. The HKCM magnets appear to have a smaller angular variation that the other small magnets. The angular varation of type HK7Nd35N cannot be measured accurately by our method. The variation of the angular variation of the big Supermagnete magnet ( $\mathrm{Su} 45 \mathrm{Nd} 45 \mathrm{NCN})$ was assesed only on 10 magnets. It appears however that the variation is well below our measurement uncertainty.

Figure 11 shows the offset angle from the same magnet, which has been measured 50 times resulting in a standard deviation of $0.645^{\circ}$.

Table VII and VIII summarize the measured angle and magnetization variations with confidence intervals.

Figures 12 shows the raw measured angular variations for the series of commercial magnets investigated.

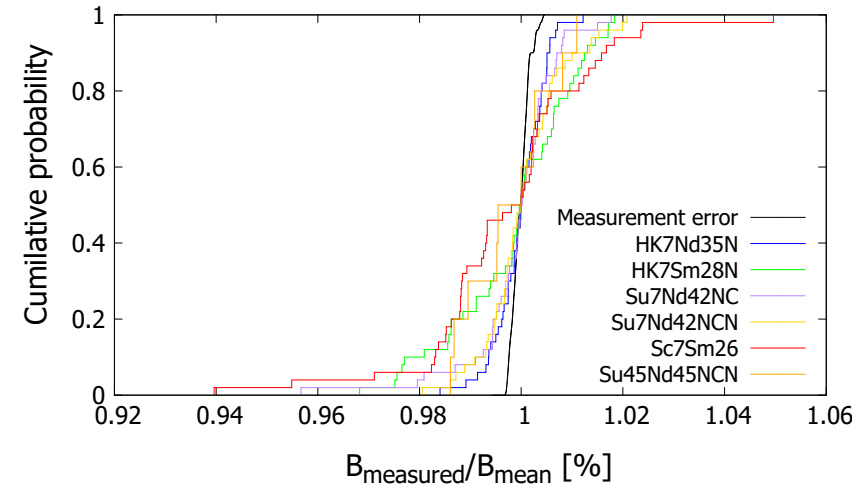

Fig. 9. Measured cumulative distribution of the residual flux density for a range of commercial magnets. The black line indicates our sensitivity limit. The sensitivity limit has been obtained from measuring 50 times the same magnet, indicated by the black line. We measured 10 different magnets with a diameter of $d=45 \mathrm{~mm}$ and a height of $h=30 \mathrm{~mm}$ (orange), and 50 magnets with a size of $7 \mathrm{~mm} \times 7 \mathrm{~mm} \times 7 \mathrm{~mm}$ for each of the other kinds of material or manufacturer. On average, commercial magnets have a magnetization variation of less than $1 \%$.

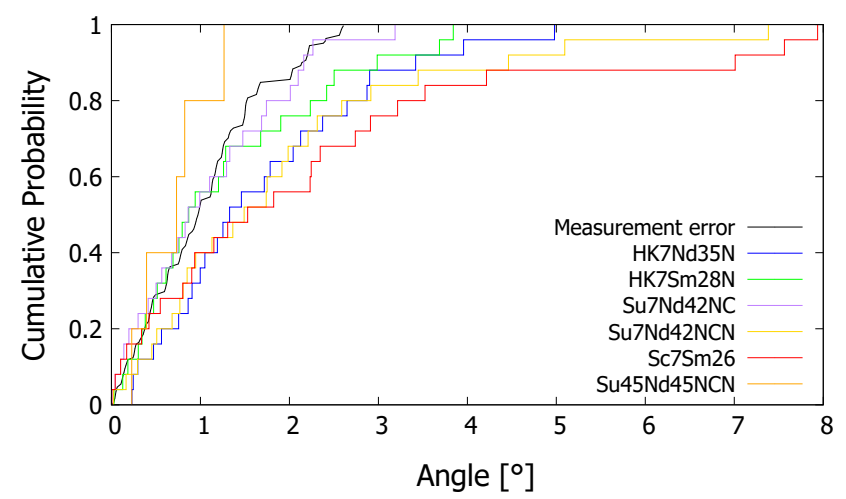

Fig. 10. Measured cumulative distribution of the field direction with respect to the $z$-axis ( $\phi$ in figure 7 ) for a range of commercial magnets. On average, commercial magnets have a field direction variation of less than $1^{\circ}$.

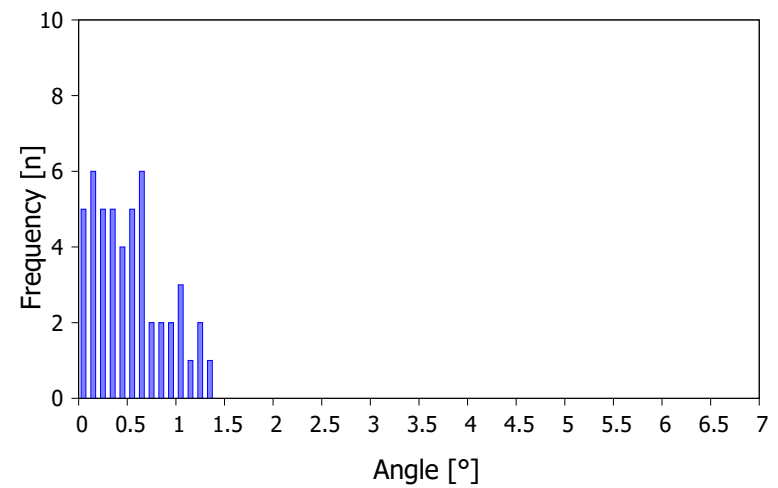

Fig. 11. The offset angle from the same magnet has been measured 50 times resulting in a standard deviation of $0.645^{\circ}$. 

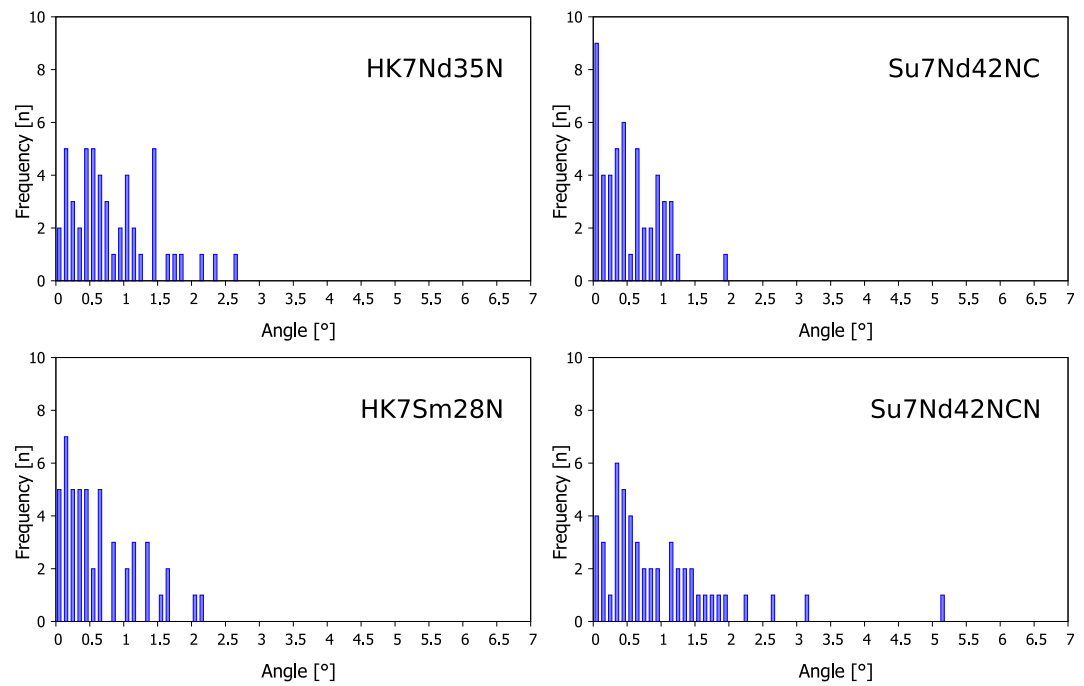

Angle $\left[{ }^{\circ}\right]$
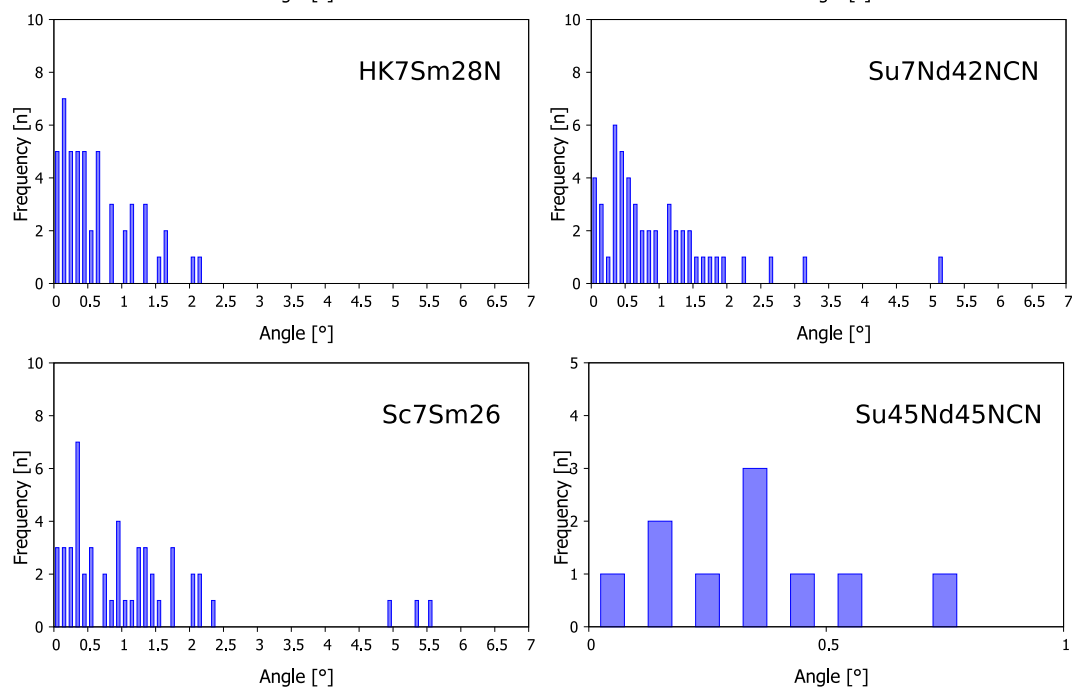

Fig. 12. Measured field angles for the range of commercial magnets investigated

TABLE VII

MEASURED ANGLE VARIATIONS.

\begin{tabular}{|c|c|c|c|c|c|}
\hline & \multirow[b]{2}{*}{ Material } & \multirow[b]{2}{*}{ Coating } & \multirow[b]{2}{*}{$\operatorname{std} \rho\left[^{\circ}\right]$} & \multicolumn{2}{|c|}{ confidence interval } \\
\hline & & & & $95 \%$ & $68.27 \%$ \\
\hline Measurement error & NdFeB N45 & $\mathrm{Ni}-\mathrm{Cu}-\mathrm{Ni}$ & 0.65 & 0.11 & 0.06 \\
\hline Supermagnete & $\mathrm{NdFeB} \mathrm{N42}$ & $\mathrm{Ni}-\mathrm{Cu}-\mathrm{Ni}$ & 1.33 & 0.2 & 0.11 \\
\hline Supermagnete & $\mathrm{NdFeB} \mathrm{N42}$ & $\mathrm{Ni}-\mathrm{Cu}$ & 0.69 & 0.113 & 0.06 \\
\hline $\mathrm{HKCM}$ & NdFeB N35 & $\mathrm{Ni}$ & 1.05 & 0.17 & 0.09 \\
\hline $\mathrm{HKCM}$ & Sm2Co17 YXG28 & $\mathrm{Ni}$ & 0.85 & 0.14 & 0.07 \\
\hline $\begin{array}{l}\text { Schallenkammer Magnet- } \\
\text { systeme }\end{array}$ & $\mathrm{Sm} 2 \mathrm{Co} 17 \mathrm{YXG}-26 \mathrm{H}$ & - & 1.72 & 0.28 & 0.14 \\
\hline Supermagnete (Cylinder) & $\mathrm{NdFeB} \mathrm{N45}$ & $\mathrm{Ni}-\mathrm{Cu}-\mathrm{Ni}$ & 0.42 & 0.13 & 0.07 \\
\hline
\end{tabular}

TABLE VIII

MEASURED MAGNETIZATION VARIATIONS.

\begin{tabular}{lllrr}
\hline \hline & Material & Coating & B [T] & $\begin{array}{c}\sigma \\
\text { confidence }\end{array}$ \\
\hline Measurement error & NdFeB N42 & Ni-Cu-Ni & 0.5425 & 0.1709 \\
Supermagnete & NdFeB N42 & Ni-Cu-Ni & 0.0002 \\
Supermagnete & NdFeB N42 & Ni-Cu & 0.4196 & 0.8631 \\
HKCM & NdFeB N35 & Ni & 0.5187 & 0.7965 \\
HKCM & Sm2Co17 YXG28 & Ni & 0.4503 & 0.9208 \\
Schallenkammer Magnet- & Sm2Co17 YXG-26H & - & 0.4362 & 0.0669 \\
systeme & & & 0.3852 & 1.2024 \\
Supermagnete (Cylinder) & NdFeB N45 & Ni-Cu-Ni & 0.0773 \\
\hline \hline
\end{tabular}


APPENDIX B

DESIGN OPTIMISATION

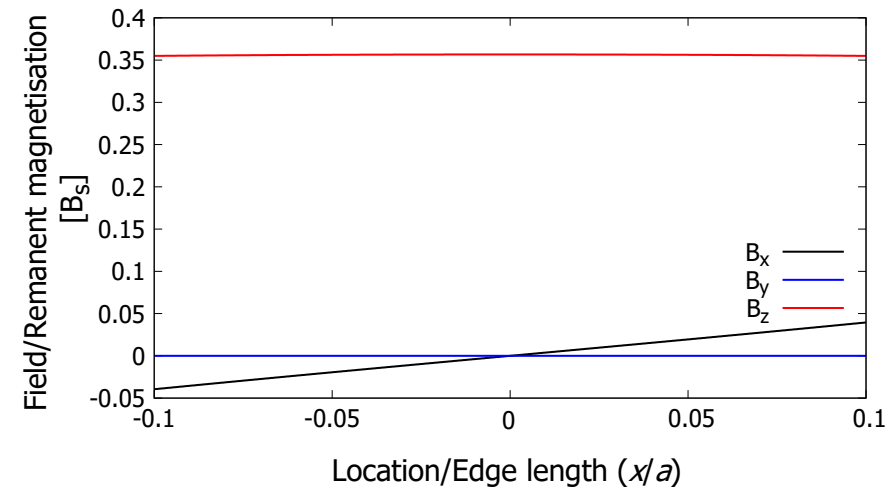

Fig. 13. Magnetic field $(x, y, z)$ above a cuboid magnet with the edge length $a$ along the $x$-axis at a distance of $0.1 a$.

Fig. 13 shows that for a cubic magnet, magnetized in the $z$-direction, there is no field in the $y$-direction $\left(B_{y}\right)$ along the $x$-axis at a distance of $0.1 a$ from the surface. The field $B_{x}$ is zero in the centre of the magnet and rises linearly with a slope of $0.04 B_{\mathrm{s}} / a$ in the positive $x$-direction, with $B_{\mathrm{S}}$ the saturation magnetization of the magnet material $[\mathrm{T}]$. The field $B_{z}$ is $0.357 B_{\mathrm{s}}$ in the centre which drops to $0.355 B_{\mathrm{s}}$ at $x=0.1 a$.

In tables X to XII the sensitivity matrix of $B_{x}, B_{y}, B_{z}$ above a cuboid magnet with the edge length $d$ along $x$-axis at a distance of $0.1 d$ is shown.

An indication of why the cuboid configuration has a much higher standard deviation than the pseudo-Halbach configuration can be seen from the sensitivity matrices of the $z$ field. We chose to show how the field in the centre and at $x=d / 2$ changes for a magnetization difference of $1 \%$ and an offset magnetization direction of $1^{\circ}$ each in the direction which creates the highest field difference at both locations. The Halbach configuration consists of 8 magnets: 4 corner magnets, 2 at the side, and 1 each on top and bottom. Adding up the sensitivity values of all the magnets results in a difference of $314 \mathrm{ppm}$ between the $z$-field at $x=0$ and $x=d / 2$. The cuboid shows a significantly higher difference of $1970 \mathrm{ppm}$. 
TABLE IX

SENSITIVITY MATRIX OF THE MAGNETIC FIELD $(x, y, z)$, GIVEN IN THE CHANGE OF $B_{S}$ AT THE SAME POSITION IN [\%] ABOVE A CUBOID MAGNET WITH THE EDGE LENGTH $a$ ALONG THE $x$-AXIS AT A DISTANCE OF $0.1 a$. VARIATIONS IN THE MAGNETIZATION ANGLE AND TILTING THE MAGNET PERPENDICULAR TO THE SIMULATED AXIS AFFECT THE MAGNETIC FIELD SIGNIFICANTLY. PLACEMENT ERRORS HAVE AN INFLUENCE IF PARALLEL TO THE FIELD. VARIATIONS IN THE DIMENSIONS HAVE A MINOR EFFECT.

\begin{tabular}{|c|c|c|c|c|c|c|c|}
\hline & \multirow[b]{2}{*}{ Variation } & \multicolumn{2}{|c|}{$B_{x}$} & \multicolumn{2}{|c|}{$B_{y}$} & \multicolumn{2}{|c|}{$B_{z}$} \\
\hline & & $x=0$ & $x=0.1 a$ & $x=0$ & $x=0.1 a$ & $x=0$ & $x=0.1 a$ \\
\hline$M$ & $1 \%$ & 0.00 & 1.10 & 0.00 & 0.00 & 1.00 & 0.99 \\
\hline tilt $x$ & $1^{\circ}$ & 0.00 & 0.00 & -0.61 & -0.62 & 0.00 & 0.00 \\
\hline tilt $y$ & $1^{\circ}$ & 0.61 & 0.51 & 0.00 & 0.00 & 0.00 & -0.48 \\
\hline$\phi\left(\theta=0^{\circ}\right)$ & $1^{\circ}$ & 0.00 & 0.00 & 0.00 & 0.00 & 0.00 & 0.00 \\
\hline$\phi\left(\theta=90^{\circ}\right)$ & $1^{\circ}$ & -0.87 & -0.88 & -0.87 & -0.86 & 0.00 & 0.19 \\
\hline$x$ & $0.1 a$ & -1.09 & -1.15 & 0.00 & 0.00 & 0.00 & 0.10 \\
\hline$y$ & $0.1 a$ & 0.00 & 0.00 & 1.09 & 1.07 & 0.00 & 0.00 \\
\hline$z$ & $0.1 a$ & 0.00 & -0.10 & 0.00 & 0.00 & -2.17 & -2.22 \\
\hline height & $0.1 a$ & 0.00 & 0.02 & 0.00 & 0.00 & 0.23 & 0.23 \\
\hline depth & $0.1 a$ & 0.00 & -0.18 & 0.00 & 0.00 & -0.01 & 0.01 \\
\hline width & $0.1 a$ & 0.00 & 0.05 & 0.00 & 0.00 & -0.01 & -0.01 \\
\hline
\end{tabular}

TABLE X

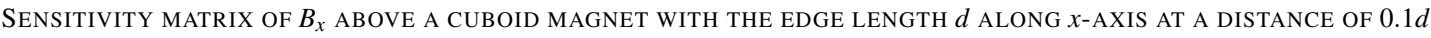

\begin{tabular}{lccccccccc}
\hline \hline$B_{x}(x)$ & $-0.1 d$ & $-0.075 d$ & $-0.05 d$ & $-0.025 d$ & 0 & $0.025 d$ & $0.05 d$ & $0.075 d$ & $0.1 d$ \\
{$\left[10^{-6} B_{\mathrm{S}}\right]$} & & & & & & & & & \\
\hline$B_{x}$ & -39520.0 & -29365.0 & -19448.0 & -9685.3 & 0 & 9685.3 & 19448.0 & 29365.0 & 39520.0 \\
$\Delta M$ & -394.6 & -294.1 & -194.8 & -96.9 & 0 & 96.9 & 194.8 & 294.1 & 394.6 \\
$\Delta$ tilt $x$ & 0 & 0 & 0 & 0 & 0 & 0 & 0 & 0 & 0 \\
$\Delta$ tilt $y$ & 1822.1 & 1972.9 & 2086.0 & 2148.8 & 2174.0 & 2148.8 & 2086.0 & 1972.9 & 1822.1 \\
$\Delta \phi=1^{\circ}, \theta=0^{\circ}$ & 0 & 0 & 0 & 0 & 0 & 0 & 0 & 0 & 0 \\
$\Delta \phi=1^{\circ}, \theta=90^{\circ}$ & -3129.0 & -3116.5 & -3116.5 & -3116.5 & -3116.5 & -3116.5 & -3116.5 & -3116.5 & -3129.0 \\
$\Delta x$ & -4121.8 & -4008.7 & -3933.3 & -3883.0 & -3870.4 & -3883.0 & -3933.3 & -4008.7 & -4121.8 \\
$\Delta y$ & 0 & 0 & 0 & 0 & 0 & 0 & 0 & 0 & 0 \\
$\Delta z$ & 360.7 & 257.6 & 165.9 & 81.6 & 0 & -81.6 & -165.9 & -257.6 & -360.6 \\
$\Delta$ height & -88.7 & -66.8 & -44.7 & -22.4 & 0 & 22.4 & 44.7 & 66.8 & 88.7 \\
$\Delta$ depth & 647.2 & 478.8 & 315.4 & 157.1 & 0 & -157.1 & -315.4 & -478.8 & -647.2 \\
$\Delta$ width & -182.2 & -137.0 & -91.4 & -45.7 & 0 & 45.7 & 91.4 & 137.0 & 182.2 \\
\hline \hline
\end{tabular}

TABLE XI

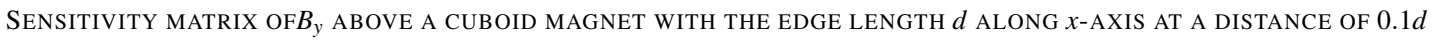

\begin{tabular}{lccccccccc}
\hline \hline$B_{y}(x)$ & $-0.1 d$ & $-0.075 d$ & $-0.05 d$ & $-0.025 d$ & 0 & $0.025 d$ & $0.05 d$ & $0.075 d$ & $0.1 d$ \\
{$\left[10^{-6} B_{\mathrm{S}}\right]$} & & & & & & & & & \\
\hline$B_{y}$ & 0 & 0 & 0 & 0 & 0 & 0 & 0 & 0 & 0 \\
$M$ & 0 & 0 & 0 & 0 & 0 & 0 & 0 & 0 & 0 \\
tilt $x$ & -2211.7 & -2199.1 & -2186.5 & -2174.0 & -2174.0 & -2174.0 & -2186.5 & -2199.1 & -2211.7 \\
tilt $y$ & 0 & 0 & 0 & 0 & 0 & 0 & 0 & 0 & 0 \\
$\Delta \phi=1^{\circ}, \theta=0^{\circ}$ & -3066.2 & -3091.3 & -3103.9 & -3103.9 & -3116.5 & -3103.9 & -3103.9 & -3091.3 & -3066.2 \\
$\Delta \phi=1^{\circ}, \theta=90^{\circ}$ & 0 & 0 & 0 & 0 & 0 & 0 & 0 & 0 & 0 \\
$\Delta x$ & 0 & 0 & 0 & 0 & 0 & 0 & 0 & 0 & 0 \\
$\Delta y$ & 3807.6 & 3832.7 & 3857.9 & 3870.4 & 3870.4 & 3870.4 & 3857.9 & 3832.7 & 3807.6 \\
$\Delta z$ & 0 & 0 & 0 & 0 & 0 & 0 & 0 & 0 & 0 \\
$\Delta$ height & 0 & 0 & 0 & 0 & 0 & 0 & 0 & 0 & 0 \\
$\Delta$ depth & 0 & 0 & 0 & 0 & 0 & 0 & 0 & 0 & 0 \\
$\Delta$ width & 0 & 0 & 0 & 0 & 0 & 0 & 0 & 0 & 0 \\
\hline \hline
\end{tabular}


TABLE XII

SENSITIVITY MATRIX of $B_{z}$ ABOVE A CUBOID MAGNET WITH THE EDGE LENGTH $d$ ALONG $x$-AXIS AT A DistANCE OF $0.1 d$

\begin{tabular}{lccccccccc}
\hline \hline $\begin{array}{l}B_{z}(x) \\
{\left[10^{-6} B_{\mathrm{s}}\right]}\end{array}$ & $-0.1 d$ & $-0.075 d$ & $-0.05 d$ & $-0.025 d$ & 0 & $0.025 d$ & $0.05 d$ & $0.075 d$ & $0.1 d$ \\
\hline$B_{z}$ & 354950 & 355720 & 356250 & 356550 & 356660 & 356550 & 356250 & 355720 & 354950 \\
$M$ & 3543.7 & 3556.3 & 3556.3 & 3568.8 & 3568.8 & 3568.8 & 3556.3 & 3556.3 & 3543.7 \\
tilt $x$ & 0 & 0 & 0 & 0 & 0 & 0 & 0 & 0 & 0 \\
tilt $y$ & 1696.5 & 1269.2 & 844.5 & 422.2 & 0 & -422.2 & -844.5 & -1269.2 & -1696.5 \\
$\Delta \phi=1^{\circ}, \theta=0^{\circ}$ & 0 & 0 & 0 & 0 & 0 & 0 & 0 & 0 & 0 \\
$\Delta \phi=1^{\circ}, \theta=90^{\circ}$ & -689.9 & -512.7 & -339.3 & -169.6 & 0 & 169.6 & 339.3 & 512.7 & 689.9 \\
$\Delta x$ & -360.7 & -257.6 & -165.9 & -81.6 & 0 & 81.6 & 165.9 & 257.6 & 360.7 \\
$\Delta y$ & 0 & 0 & 0 & 0 & 0 & 0 & 0 & 0 & 0 \\
$\Delta z$ & -7929.4 & -7841.4 & -7778.6 & -7753.5 & -7740.9 & -7753.5 & -7778.6 & -7841.4 & -7929.4 \\
$\Delta$ height & 821.8 & 826.9 & 830.6 & 833.2 & 833.2 & 833.2 & 830.6 & 826.9 & 821.8 \\
$\Delta$ depth & 40.0 & 8.4 & -13.2 & -25.8 & -30.0 & -25.8 & -13.2 & 8.4 & 40.0 \\
$\Delta$ width & -33.0 & -31.7 & -30.7 & -30.2 & -30.0 & -30.2 & -30.7 & -31.7 & -33.0 \\
\hline \hline
\end{tabular}




\section{APPENDIX C \\ MEASUREMENTS}

Figure 14 shows the measured magnetic field $\left(B_{z}\right)$ of Cuboid and Pseudo-Halbach configuration along the $x$-axis for $d=$ $8 \mathrm{~mm}$. Table XIII shows the homogeneities of the measured configurations.

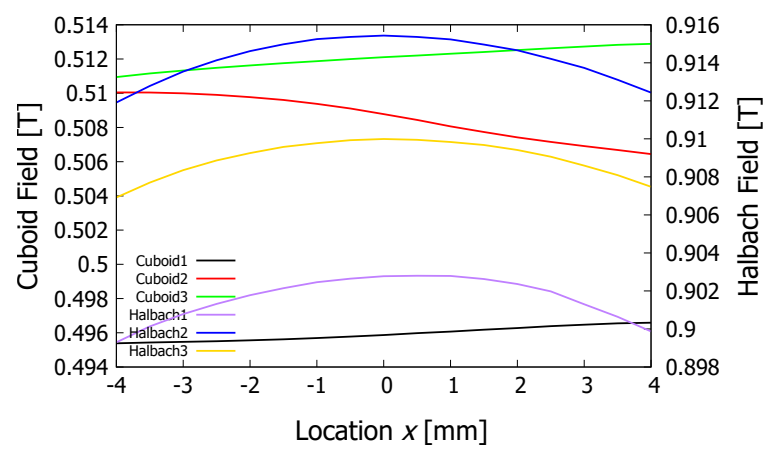

Fig. 14. Measured magnetic field $(z)$ of Cuboid and Pseudo-Halbach configuration along $x$-axis.

TABLE XIII

Measured homogeneity of CUBOID AND PSEUdo-HALBaCH CONFIGURATIONS

\begin{tabular}{lr}
\hline \hline & $\begin{array}{c}\text { Inhomogeneity } \\
{[\mathrm{ppm}]}\end{array}$ \\
\hline Cuboid 1 & $748(3)$ \\
Cuboid 2 & $2250(3)$ \\
Cuboid 3 & $1021(3)$ \\
Pseudo-Halbach 1 & $1088(3)$ \\
Pseudo-Halbach 2 & $1081(3)$ \\
Pseudo-Halbach 3 & $929(3)$ \\
\hline \hline
\end{tabular}

